\title{
LA INSPECCIÓN EDUCATIVA VISTA DESDE LA ESCUELA
}

\section{Educational Inspectorate from the School point of view}

\author{
Alexandre Camacho Prats \\ Govern de les Illes Balears \\ Correo-e: alexcprats@hotmail.com \\ Recibido: 30 de junio de 2018 \\ Envío a informantes: 8 de julio de 2018 \\ Aceptación definitiva: 6 de septiembre de 2018
}

Resumen: En este artículo indagamos sobre la visión que se tiene de la Inspección Educativa desde la escuela. Para ello, hemos triangulado los datos aportados por cinco colectivos que en este estudio nos parecen determinantes: por una parte, la opinión de maestros; en segundo lugar, las consideraciones de equipos directivos de colegios públicos de educación primaria; en tercer lugar, las aportaciones de personal de administración y servicios; en cuarto lugar, los argumentos de padres, madres y tutores legales de alumnos, y, en último y destacado lugar, la visión de los propios alumnos, protagonistas principales y la verdadera razón de ser de nuestro sistema educativo. La investigación, implementada en España durante el curso escolar 2017/20I8 mediante entrevistas y la aplicación de un cuestionario, revela que a pesar de que la valoración hecha por todos los colectivos sobre la Inspección es pertinente, al entender que esta institución es importante y tiene capacidad de influir en la mejora escolar, sin embargo, evidencia la necesidad de que la Inspección vuelva a estar más en contacto con la vida escolar y menos con la burocracia del despacho.

Palabras clave: Inspección Educativa; inspectores de Educación; escuela; educación primaria; calidad de la enseñanza; mejora de la educación; impacto en la escuela.

Aвstract: In this article, we enquire on the vision about Educational Inspectorate from the school point of view. To do so, we triangulated the data provided by five groups that seem determinant in this research: on the one hand, the opinion of Primary teachers; secondly, the considerations of Elementary State School principals; third, the contributions of administration and services staff; fourth, the perceptions of student's 
parents and legal guardians and, ultimately and prominently, the vision of the students themselves, as main protagonists and the true 'raison d'être' of our educational system. The research, implemented in Spain during the school year 2017/2018 through interviews and the application of a questionnaire, reveals that although the assessment made by all groups about the Spanish Inspectorate is relevant, by understanding that this institution is important and has ability to influence school improvement, somehow evidences the need for the Inspection to be more in touch with school life and less with office bureaucracy.

KEY words: Educational Inspectorate; Education inspectors; school; Primary school; teaching quality; education improvement; impact in school.

\section{Introducción}

$\mathrm{L}$

A Inspección educativa ha estado ligada a la enseñanza primaria desde su nacimiento como cuerpo profesional en 1849. De hecho, el que es considerado como el artífice de la creación de la Inspección profesional, Antonio Gil de Zárate, ya exponía la necesidad de inspectores «para que la instrucción primaria recibiese el impulso constante que necesita, y para que las medidas dictadas en su favor no quedasen reducidas a meros e ineficaces proyectos» (Gil de Zárate, 1995 [I855]: t. I, 299). No en vano, el propio Gil de Zárate destaca el exitoso «impulso» que la Inspección había ejercido en la enseñanza primaria europea:

Son precisos funcionarios formados a propósito, dotados de conocimientos y cualidades especiales, que estén ejerciendo constantemente su importante ministerio, que hagan de esto la ocupación de su vida, que en ello funden su subsistencia, que sean responsables respecto del que los emplea, y den cuenta a menudo del desempeño de sus funciones y de los adelantos que a sus afanes se deben. Esta institución ha sido en todas partes la que mayor impulso ha dado a la instrucción primaria en Europa; y era forzoso introducirla entre nosotros, si se habían de alcanzar las mismas ventajas en punto de tanto interés para las naciones cultas (ibídem: $30 \mathrm{I}$ ).

En un escenario en el que queda claramente manifiesta y necesaria la vinculación directa de la Inspección Educativa con la enseñanza primaria desde su origen -hace ahora I70 años-, y sobre todo teniendo en cuenta que, en efecto, la Inspección ha tenido un próspero y fecundo influjo histórico en el devenir no solo de la mejora escolar, sino con ella, también de la sociedad, no obstante, cabe decir que en las últimas décadas el peso de la Inspección en la realidad cotidiana de las aulas parece haber decrecido exponencialmente. Esto ha propiciado que los inspectores de Educación estén la mayor parte de su tiempo dedicados a tareas burocráticas que, en muchos casos, son de bajo calado pedagógico y más propias de auxiliares administrativos que de inspectores funcionarios de un cuerpo tan estratégico en el sistema educativo, con un noble cometido tan impropiamente desarrollado por exigencias del guion político que ha alarmado no solo a los propios inspectores, sino a académicos estudiosos de la supervisión y a numerosos colectivos (directores, maestros, asociaciones de padres, etc.).

Son ya muchas las voces autorizadas, de autores de referencia que estudian el impacto de la Inspección en la mejora de la calidad educativa, que denuncian la situación de crisis de la institución inspectora. Alertan que la Inspección Educativa se halla, 
como dice Sarasúa (20I0), «en vía muerta», en un panorama en el que «la inspección se convierte en una entidad de carácter débil» (Castán, 20I4: 5) al estar alejada de los alumnos. Recientemente, este mismo autor ha advertido del riesgo de que se convierta en «una inspección que no inspecciona» (Castán, 20I6: 2), bien por el exceso de tareas burocráticas que derivan en la escasez de visitas de corte pedagógico, bien por el laxo o nulo impacto en la evaluación de la práctica docente o, lo que es peor, por la dependencia e injerencias políticas. Secadura (20II) aboga por la necesidad de un nuevo modelo de Inspección Educativa «que le permita tener relevancia en el ámbito educativo del futuro» (p. I), a la vez que un autor referente indiscutible como Soler Fiérrez (2015: 152) reclama que «los inspectores deben esforzarse para procurar involucrar a los profesores en un proceso de autoevaluación que se refiere a la capacidad para evaluar el trabajo que están llevando a cabo con vistas a mejorarlo». Esteban Frades (20I4) lamenta que «uno de los principales problemas de la inspección es que cada vez está más desaparecida e influye menos en el sistema educativo» (p. 264). Nosotros, en otros espacios, ya recalcamos que los inspectores de Educación están infrautilizados y desaprovechados por la Administración educativa (Camacho, 20I4) y que la visita que giran los inspectores está malherida (Camacho, 2015) por quedar reducida, en demasiadas ocasiones, únicamente al despacho del director y alejada de las aulas. En otras aportaciones académicas planteamos que la responsable de que la Inspección Educativa vuelva a ser influyente es la Administración educativa (Camacho, 20I7; Camacho y Rodríguez Bravo, 2016).

De hecho, el día io de agosto de 2017 aparecía una noticia en el diario El Mundo que expone el modo efectivo y sistemático con el que los inspectores británicos visitan las aulas, tan al contrario de lo que sucede en España, aunque los propios inspectores españoles quisieran tener más tiempo para visitar y evaluar los centros educativos en profundidad. En dicho artículo se expone que «En Gran Bretaña, los inspectores se meten en las clases, miran los deberes, los exámenes, cómo se consigue un ambiente de orden o no en cada colegio. Y, luego, su evaluación es pública. ¿En España? Nada parecido». Prosigue la noticia con las aseveraciones de una inspectora y parlamentaria autonómica de Madrid, que lamenta la situación en nuestro país a la vez que puntualiza que «todas las asociaciones reivindican más presencia en las aulas y menos papeleo».

En efecto, las principales asociaciones de inspectores de educación así lo reclaman. El día 5 de abril de 20I7, Jesús Marrodán, presidente de la Unión Sindical de Inspectores de Educación (USIE) -la primera entidad representativa de inspectores que compareció ante el Congreso de los Diputados en la subcomisión parlamentaria que se encarga de lograr un pacto estatal político y social en educación- exigía a sus señorías la necesidad de «una Inspección Educativa que, mediante el desarrollo de normativa de carácter básico, tenga autonomía técnica e independencia profesional para garantizar el ejercicio de los derechos y el cumplimiento de las obligaciones de los miembros de la comunidad educativa y de las propias administraciones vertebrando el Estado. Algo de lo que ahora se adolece ya que, si los órganos de control se politizan, estos pierden su sentido y razón de ser, relegando a los ciudadanos a mera indefensión» ${ }^{2}$. Por su parte, Carlos Utrera, presidente de la Federación ADIDE (Asociación de Inspectores de

Véase la noticia en El Mundo en http://www.elmundo.es/blogs/elmundo/mejoreducados/2017/o8/ Io/la-inspeccion-educativa-del-michaela-a.html.

Véase la nota de prensa de USIE en http://usie.es/wp-content/uploads/20I8/o6/NOTA-DE-PRENSAUSIE-NUEVA-MINISTRA-DE-EDUCACIÓN-2OI8.pdf. 
Educación, la mayoritaria en el colectivo inspector, con un 60 por ciento de los inspectores españoles) expone de manera análoga a usie sus críticas a la falta de independencia y a la politización, al reclamar más independencia de la Inspección del poder político porque, según dijo también en el Congreso de los Diputados, «con frecuencia se tiende a instrumentalizar la Inspección educativa como si fuese la guardia pretoriana de la Administración»³.

En definitiva, la Inspección Educativa tiene unas funciones y atribuciones muy claras enfocadas a la mejora de la calidad de la educación, aunque el tratamiento de la atribución más destacada de los inspectores - la visita de inspección- parece que necesita un revulsivo desde la propia Administración educativa en orden a que la Inspección educativa vuelva a ser un servicio efectivamente influyente en la escuela. Por ello, para conocer la visión que diferentes actores que configuran la escuela tienen sobre la Inspección Educativa, hemos realizado una investigación que detallamos en el siguiente epígrafe.

\section{Método}

Para examinar las impresiones que distintos sectores que conforman la escuela tienen sobre la Inspección Educativa, hemos llevado a cabo una investigación implementada durante cinco meses, de febrero a junio de 20I8. Nuestro objetivo principal era conocer la visión que se tiene de la Inspección y, por ello, hemos diseñado un estudio empírico fundamentado en dos tipos de técnicas (la entrevista y el cuestionario), aplicadas a fuentes informantes de cinco colectivos fundamentales en la escuela:
a) Maestros.
b) Equipos directivos.
c) Personal de administración y servicios (PAs).
d) Padres, madres y tutores legales de alumnos.
e) Alumnado.

El estudio empírico se realizó mediante dos técnicas (cuestionarios y entrevistas) según exponemos seguidamente.

\subsection{Cuestionarios}

Seleccionamos una muestra inicial de i2o escuelas públicas de todas las comunidades autónomas españolas elegidas aleatoriamente. Tras presentar el objetivo del estudio y solicitar la autorización pertinente a la Dirección de dichos centros para contar con su colaboración (con especial énfasis en nuestro interés en conocer de primera mano las percepciones de todos los colectivos, también el de alumnos), logramos que un total de 96 escuelas respondiesen a nuestra petición. Sin embargo, nueve de ellas han declinado participar y no han remitido sus respuestas, pese a nuestro reiterado interés. En total, hemos recibido las valoraciones de miembros de 87 colegios de once

\footnotetext{
Véase la noticia de la comparecencia de ADIDE en el Congreso de los Diputados en el diario El País (27-06-20I7): https://politica.elpais.com/politica/20I7/06/27/actualidad/I498566448_553468.html.
} 
comunidades autónomas españolas; esto representa un 72,5 por ciento de los centros de la muestra inicial, que, a nuestro entender, es un dato muy satisfactorio.

Siendo conocedores de que la organización escolar está repleta de responsabilidades y tareas diarias, queríamos no cargar con un exceso de trabajo a los colectivos participantes a la vez que lograr el máximo de respuestas por parte de todas las fuentes informantes. En consecuencia, optamos por presentar un cuestionario con tres preguntas dirigidas a los tres colectivos profesionales (maestros, equipos directivos y PAs) y una pregunta abierta a los otros dos restantes, los administrados: alumnado y sus familias.

Por cuestiones de operatividad y eficiencia, dada la distancia geográfica entre las escuelas participantes, se contactó con los distintos centros con la invitación formal a participar en el estudio y con la solicitud de trasladar las diferentes cuestiones a los colectivos pertinentes, a las que podían acceder anónimamente mediante un enlace a un cuestionario elaborado con un formulario de Google Docs. El mencionado cuestionario dirigido a los profesionales escolares está compuesto de tres únicas preguntas, dos de ellas abiertas:

I. ¿Cuál es su visión de la Inspección Educativa? (Respuesta abierta)

2. ¿Visita las aulas de la escuela con asiduidad el inspector o inspectora de Educación? (Respuestas cerradas: Sí, No, No lo sé).

3. En caso afirmativo en la pregunta 2 , ¿sabe qué aspectos se tratan en las visitas que realiza el inspector o inspectora en su escuela?

Además, se envió una pregunta abierta dirigida a los dos colectivos restantes: por una parte, padres, madres y tutores legales del alumnado y, por otra parte, al propio alumnado. Esta cuestión es la primera pregunta abierta del cuestionario anterior: «¿Cuál es su visión de la Inspección educativa? (Respuesta abierta)».

El cuestionario solicitaba no solamente la elaboración de la/s respuesta/s y su envío telemático, sino también que indicaran a qué colectivo pertenecen; esto nos ha facilitado mucho el trabajo de codificación de las respuestas obtenidas según los variados grupos informantes. Las respuestas ofrecidas por las 87 escuelas reflejan las opiniones de 247 personas de estos colectivos:

a) 130 maestros.

b) 87 directores.

c) II miembros del PAs.

d) I9 padres de alumnos.

e) No recibimos ninguna respuesta de alumnado mediante el cuestionario informático.

Cabe señalar, por supuesto, que el trabajo de gestión de los equipos directivos que participaron en el estudio en materia de traslado del enlace del cuestionario a todos los implicados de sus respectivas escuelas ha sido excelente y totalmente de agradecer.

\subsection{Entrevistas}

Por otra parte, aplicamos entrevistas a miembros de los cinco sectores mencionados que conforman la escuela. Nos decantamos por esta opción porque queríamos 
obtener datos con más profundidad de lo que ofrece un cuestionario, y tener elementos para contrastar las valoraciones efectuadas, a la vez que poder departir con fuentes informantes con más tiempo y calado. Además, pudimos acceder al colectivo del alumnado para conocer su visión sobre la Inspección Educativa. De este modo, efectuamos 63 entrevistas realizadas personalmente con informantes de ocho escuelas públicas, según especificamos seguidamente:

I. Veinte entrevistas con maestros.

2. Ocho entrevistas con directores.

3. Cinco entrevistas con PAs (dos auxiliares administrativos, un portero y dos monitoras de un comedor escolar).

4. Diez entrevistas con padres de alumnos.

5. Veinte entrevistas con alumnos de sexto de Primaria. En todas las entrevistas estuvieron presentes los maestros tutores de los alumnos, que asistieron invitados por nosotros, aunque sin voz ni voto por voluntad propia y nuestra para no condicionar las respuestas de niños y niñas.

Las entrevistas se iniciaron con una breve exposición del objeto de este estudio y con la formulación de la pregunta común «¿Cuál es su visión de la Inspección Educativa?». A ello, por supuesto, en numerosos casos se plantearon también otras cuestiones subyacentes propias de la técnica de la entrevista, que ayudaron a algunos entrevistados a exponer su visión de forma más definida.

En total, entre la aplicación de los cuestionarios y las entrevistas realizadas, hemos podido lograr informaciones de un total de 3 Io personas informantes procedentes de 95 escuelas públicas de once comunidades autónomas españolas. En todos los casos, en el estudio empírico hemos intentado respetar escrupulosamente las siguientes premisas:

- Se ha procurado el máximo rigor científico posible.

- Se ha ofrecido el máximo respeto ético posible.

- Se han solicitado los permisos pertinentes para acceder a las entrevistas con los alumnos menores.

- No ha habido interés económico: ninguna de las partes ha percibido compensación alguna en absoluto, más allá de compartir información y datos para la presente investigación.

- Se ha mantenido la necesaria confidencialidad de los datos de todas las instituciones y personas participantes.

\section{Resultados}

El análisis de las respuestas ofrecidas tanto en los cuestionarios como en las entrevistas ofrece resultados que sintetizamos en relación con los grupos participantes.

Las respuestas de los maestros apuntan claramente a que la percepción que actualmente tienen de la Inspección Educativa no es positiva. En general, afirman que saben que los inspectores e inspectoras de Educación desarrollan una labor intensa, aunque lamentan que no sea en contacto directo con ellos. Revelan que están desconectados de la escuela y las evidencias son prácticamente unánimes: un maestro veterano recuerda que «hace muchos años, los inspectores venían al aula, pasaban horas con 
nosotros y nos hacían recomendaciones pedagógicas que nos resultaban muy útiles, pero ya no se pasan por las aulas, y creo que deberían hacerlo para ayudar a mejorar la enseñanza, es una lástima pero no vienen a ver las clases ni a hablar con nosotros. Quizás alguno habrá, pero en general, no vienen». Una maestra sostiene que «seguro que tienen mucho trabajo, pero por las clases no se les ve». Otro maestro afirma que «seguro que hacen muchas cosas y andarán ocupadísimos en no sé qué, pero no siento que me ayuden en nada», a la vez que otro docente argumenta que «la verdad, y esto lo he experimentado en otros centros, los inspectores cuando vienen es porque hay lío con algo o porque les ha llamado el director, y si no eres el implicado, ni te enteras de que ha estado en el centro porque están con el director y se van enseguida». Otra maestra apunta que a pesar de que no ha recibido la visita de ningún inspector, le hubiera gustado para recibir retroalimentación sobre su práctica docente: «Nunca, en mi vida profesional, he visto a un inspector entrar en mi clase, y llevo 27 años de docencia. Estaría bien que viniesen porque así tendría una opinión fundada de cómo lo hago... pero nada, de verdad lo digo, nunca han venido». Otra maestra comparte impresiones al lamentar que «mi visión ni es buena ni mala, porque no he tenido el gusto de recibirlos. Y es un tema que a veces he hablado con mis compañeras, no me importaría que me evaluasen si eso me permite mejorar. Además, igual algún maestro tóxico dejaría de serlo si los inspectores vieran lo que hacen algunos». Numerosos maestros critican que los inspectores son «burócratas» y una maestra dijo que «están en sus despachos, muy alejados de la primera línea, y cuando digo primera línea me refiero, ya sabes, al aula, al contacto con los profesores y los críos. Eso no lo hacen». Otra maestra se pregunta «si mañana desaparece la Inspección, todo seguiría igual, los centros seguiríamos igual sin ellos». Otra maestra sostiene que «los inspectores son enviados del gobierno de turno y no sé qué pintan más allá de que controlan, porque... ¿ ‘tienen alguna misión pedagógica? Es que no lo sé...».

El colectivo de directores escolares tiene un punto de vista diferente al de los maestros participantes en el estudio. El 89,5 por ciento de los directores informantes del estudio expresan que la Inspección Educativa les ayuda en muchas ocasiones. Un director argumenta que «siempre que lo he necesitado ha estado ahí para resolver mis dudas». Otro director expone que «mi visión es buena, porque cuando necesito a mi inspectora es muy receptiva y sabe ayudarme». Otro director explica que «saben lo que están haciendo en muchos otros centros y nos asesora bastante. Además, en temas de normativa me ha explicado algunos puntos importantes y, a decir verdad, me va bien cuando el inspector dice que algo se tiene que hacer, porque eso para mí es un aval en el claustro, nadie se atreve a cuestionarlo». Una directora dice que «me han salvado de más de un problema gordo» y otra directora indica que «tienen demasiados centros que atender, pero por lo general mi visión es buena». Otro director, sin embargo, sostiene que «normalmente me han ayudado, pero no me gusta cuando viene el inspector con aires de que es mi jefe». Una directora dice que «cuando viene es porque lo he llamado y muchas veces ni viene, porque lo tratamos por teléfono». Otra directora indica que «en toda mi carrera he tenido a nueve o diez inspectores y cada uno era diferente, cada uno tiene sus manías. Incluso una vez uno que llegó me hizo rectificar algo que el anterior me había obligado a hacer... pero a grandes rasgos, me han asesorado bien si lo precisaba, querían saber algunos datos para sus estadísticas y poco más».

El colectivo del personal de administración y servicios expone de modo unánime que su visión es buena, si bien es cierto que la percepción es de un perfil muy 
jerárquico. Tal como dice una auxiliar administrativo, la visita del inspector es que «viene el jefazo». Otra auxiliar administrativo también dice que «cuando llama o viene la inspectora, todos se cuadran, y la directora la que más». Sostienen mayoritariamente que desconocen qué aspectos abordan cuando vienen al centro, porque con ellos no tratan ningún tema, pues, tal como dice otro miembro del pas de una escuela, «si han venido alguna vez, ha sido a ver al equipo directivo y se encierran en un despacho juntos». Ninguno expuso que supiera si los inspectores visitan las aulas.

Los padres, madres y tutores legales de alumnos afirman de modo muy unánime que su visión es positiva. Una madre sostiene que «he hablado alguna vez con el inspector porque soy miembro del consejo escolar de la escuela de mis hijas y ha venido a reunirse con nosotros en diversas ocasiones, me parece que se preocupa de que se cumpla lo que toca, mi visión es buena». Otra madre indica que «no sé qué temas específicos tratan en la escuela cuando va el inspector, se lo tendrías que pedir a ellos, pero lo que sé es que cuando el curso pasado unos padres fuimos a ver al inspector por un problema con el centro y fue muy amable, se mostró preocupado y resolvió la cuestión rápidamente. Y puedo decir tranquilamente que el problema se resolvió bien para todos, cosa que no es fácil». Otro padre se congratula porque «sé que el inspector va a la escuela porque mi hijo me lo comenta» y en este caso constatamos que corrobora la visita a las aulas, pues continua diciendo que «me dijo mi hijo que en varias ocasiones el inspector ha ido a su clase, se ha quedado un buen rato mientras el profesor impartía su clase y luego hablaban».

Finalmente, el sector de los alumnos sostiene visiones contrapuestas. Algunos dicen que han visto a inspectores en sus aulas: un alumnos nos explicó que a veces han estado "en la clase y hablaban con la profesora», o por la escuela, pues argumenta que «nos dicen que está el inspector, o que mañana vendrá y todos tenemos que portarnos bien». Otro alumno explicaba que "ayer mismo vino un inspector a la clase porque nos hacían unos exámenes y estaba con nosotros mientras los hacíamos»; sin embargo, otros manifiestan que no han compartido tiempo ni espacio con inspectores: una alumna dijo que «nunca he visto a un inspector», y otra afirmó que «que yo sepa, en mi clase nunca ha entrado un inspector».

\section{Conclusiones y discusión}

El objeto de esta investigación era conocer la visión que se tiene desde la escuela sobre la Inspección Educativa y, tras el análisis de los datos resultantes, podemos establecer diversas conclusiones al respecto.

a) Hay varios colectivos que manifiestan con rotundidad que los inspectores e inspectoras no visitan las aulas como se espera de ellos, a pesar de que esta es la atribución más destacada de la Inspección educativa y la que les permite conocer y comprobar in situ la realidad escolar para tratar de contribuir a su mejora. De hecho, los colectivos que expresan que la Inspección no visita las clases con frecuencia son, sobre todo, los dos que están en las aulas: maestros y alumnos.

b) Los maestros lamentan que no reciben retroalimentación constructiva de su acción docente de parte de un colectivo tan facultado y autorizado como los inspectores de Educación. Asimismo, han dicho que hace muchos años recibían visitas de oficio y que no se presentan desde hace tiempo en sus aulas; de hecho, alguno 
reprochó que en toda su carrera profesional no haya recibido la visita de un inspector. Acontecimientos como estos no contribuyen a forjar una percepción optimista sobre la labor de la Inspección, sino que tejen una visión negativa y estéril de la función inspectora. No obstante, hay una visión expuesta por maestros que entienden que los inspectores suponen un activo en la escuela, al verles capaces de ofrecer un influjo beneficioso en los docentes y discentes, pues lamentan que no les visiten: el mero hecho de criticar su ausencia en las aulas puede entenderse como un lamento por no poder obtener un halo de mejora que emana de un cuerpo de funcionarios expertos en educación y con la condición de autoridad pública en el ejercicio de sus funciones, a pesar de que los maestros saben que los inspectores no acuden a las aulas porque se están ocupando de multitud de tareas burocráticas que los alejan de los centros.

c) La visión que tienen los directores es positiva, al ver la Inspección como una ayuda y una garantía ante muchos de sus problemas. Afirman que valoran mucho la función asesora de la Inspección, pero a pesar de que expresan que confían en ella de un modo prácticamente unánime (cerca del 90 por ciento), cabe señalar que indican que la visita que giran los inspectores a sus respectivos centros, habitualmente, se suele limitar al despacho con ellos o, en muchas ocasiones, lo solventan sin visita presencial, mediante llamadas telefónicas. Esto corrobora la visión que tienen maestros y alumnos de que la Inspección no inspecciona en las aulas.

d) El colectivo del Pas tiene una visión muy jerárquica de la figura del inspector; lo tildan de «jefazo» y que con su mera presencia en la escuela «todos se cuadran», incluso si no acude y decide realizar una llamada telefónica. Sin embargo, también podemos concluir que este colectivo respalda la visión de que la Inspección no entra en las aulas con asiduidad, pues, bien afirman que no lo saben, o bien dicen que tratan los asuntos en el despacho encerrados con el equipo directivo.

e) Los padres, madres y tutores legales manifiestan una visión positiva de modo unánime. Ven que la Inspección Educativa es garante de la legalidad y de velar por los derechos de sus hijos, pues expresan que les ofrecen ayuda y asesoramiento, y son capaces de mediar en conflictos que afectan a sus hijos en los centros docentes. Aun así, apoyan sus aseveraciones fundamentalmente en las visitas que ellos mismos como padres han hecho en las sedes de la Inspección para reunirse con el inspector en su despacho y no tanto en las visitas que el inspector haya girado a la escuela. Sin embargo, si bien son muy escasas, también hay alguna afirmación que avala que la Inspección va a las aulas en las que estudian sus hijos, pues ellos mismos lo cuentan a sus progenitores.

f) El sector del alumnado tampoco deja claro un plan de visitas de la Inspección efectuado de manera recurrente a las aulas. Algún alumno entrevistado dijo que ha compartido con inspectores en el aula, en pruebas de evaluación, o hablando con los docentes, etc., aunque también hay informantes que sostienen que no han visto a un inspector de Educación en su vida.

g) Podemos concluir, también, que los autores de referencia que hemos detallado en la Introducción señalan que urge una revisión de la visita de inspección en orden a que se centre en la calidad de la enseñanza, en los procesos educativos, en la supervisión de la acción docente, etc., y que, para que esto sea posible, los inspectores deben poder visitar las aulas, al ser el alumnado el principal actor de la educación, a la vez que el profesorado es clave en todos los procesos educativos. 
h) También concluimos que la Inspección Educativa no puede desarrollar plenamente sus funciones si su actuación queda limitada. Esto es una debilidad y una potencial amenaza de nuestro sistema educativo, cuando debería ser una fortaleza clara y evidente.

i) Concluimos, también, que el propio colectivo de inspectores de Educación desea gozar de más tiempo para poder realizar sus funciones adecuadamente, con especial referencia a la atribución de la visita, para que sea en efecto en contacto directo, sistemático y preferente con los docentes y los alumnos, así como también supervisando los espacios, planes, programas, servicios y demás elementos que puedan coadyuvar a que el influjo de la Inspección tenga un impacto beneficioso en la mejora de la calidad de la enseñanza. Las asociaciones y sindicatos profesionales de inspectores lo han recalcado muy claramente y exigen que la Administración ofrezca un marco de actuación que fomente el adecuado desempeño de su labor en el sistema educativo para que vuelva a ser estratégica y útil.

En definitiva, esta investigación pone en relieve que la comunidad educativa no aprecia una presencia de los inspectores e inspectoras en las aulas de modo sistemático y recurrente, con la intención de que la Inspección pueda llevar a cabo sus funciones con un perfil pedagógico en verdadero contacto con los actores principales de la educación. Además, esta reclamación procede también de la Inspección Educativa, que exige poder liberarse de cargas burocráticas excesivas impuestas que apartan a los inspectores e inspectoras del contacto diario y presencial con maestros y alumnos en las escuelas.

Por supuesto, se constata que desde la escuela reclaman la presencia de la Inspección en las aulas, al ver que puede ayudar a mejorar la calidad de la educación. Sin embargo, parece que la propia Inspección educativa - primera interesada en poder tener menos cargas para visitar más y mejor los centros que deben supervisar- no tiene la suficiente entidad para solventar esta problemática, pues está sujeta a un plan de trabajo establecido por los responsables políticos de la Administración educativa. En consecuencia, solamente teniendo en cuenta la capacidad de impacto beneficioso y el influjo pedagógico que se está desaprovechando diariamente, al tener a estos funcionarios altamente cualificados sin que puedan hacer su noble labor como de ellos se espera, se podrá analizar el alcance de la acción inspectora y la debilitada visión que de ellos se tiene. Paradójicamente, justo cuando estamos en un escenario social que los necesita tanto para aprovechar todo su potencial, experiencia y saber, la Administración, que debería promover su presencia diaria en las clases para que colaboren en la mejora de la vida de las escuelas y, por ende, de la vida de las personas, los mantiene lejos de la realidad de las aulas, sin que puedan influir en el adecuado desarrollo del profesorado y del alumnado.

Pensamos que no se puede permitir un modelo de actuación de la Inspección Educativa tan alejado de la escuela y basado en visitas incidentales, porque no solo no colabora en su mejora didáctica y pedagógica, sino que, apartándose de su seno más interno, promueve percepciones que están lejos de ser positivas y, por supuesto, se acentúa más la sensación de crisis y de falta de credibilidad en la institución. Por ello, consideramos que urge una revisión del modelo de Inspección Educativa para que vuelva a ser la institución creíble y promotora del mejor impacto educativo en todos los miembros que configuran la organización escolar. La Inspección Educativa debe 
volver a supervisar directamente en las aulas de un modo preferente, pues desde la escuela se reclama su presencia a la vez que la razón de ser de la Inspección implica su presencia en la escuela para que pueda ayudar en su mejora constante, haciendo de esta atribución la principal misión de los inspectores e inspectoras. Solo así podrán favorecer la calidad de la enseñanza y la mejora misma de la propia Inspección. Así lo han hecho históricamente, cuando más impacto beneficioso han tenido en el sistema educativo y, con él, en el conjunto de la sociedad. Así ha sido y no debe dejar de serlo.

\section{Bibliografía}

Camacho, A. (2014) Funciones y quehaceres de los inspectores de Educación en Baleares. Un estudio de casos. Tesis doctoral. Facultad de Pedagogía, Universidad de Barcelona. https:// www.tdx.cat/bitstream/handle/10803/I34797/ACP_TESI.pdf?sequence=I.

Camacho, A. (20I5) La visita de inspección, su función malherida. REICE, Revista Iberoamericana sobre Calidad, Eficacia y Cambio en Educación, I3 (4), 79-9I. http://www.rinace.net/ reice/numeros/arts/voliznum4/arts.htm.

Camacho, A. y Rodríguez Bravo, M. (2016) La Inspección educativa influyente, un reto para la Administración educativa. En J. L. Bernal (coord.) XIV Congreso Interuniversitario de Organización de Instituciones Educativas CIOIE "Globalización y organizaciones educativas» (pp. 257-265). Dpto. de Ciencias de la Educación, Universidad de Zaragoza. http://www.unizar.es/cce/documents/ciole.pdf.

CAmacho, A. (2017) Empowering Education Inspectorate of Spain: a real challenge. Conferencia leída en la British Educational Leadership, Management and Administration Society, Belmas, 2017 International Conference (7-9 julio). Stratford-upon-Avon, Reino Unido.

Castán, J. L. (20I4) El alumno, referente de la Inspección educativa. Avances en Supervisión Educativa, núm. 2I. https://avances.adide.org/index.php/ase/article/view/86/85.

CAstán, J. L. (2016) La función docente y la función inspectora, retos para la mejora en una sociedad cambiante. XVII Encuentro Nacional de Inspectores de Educación, UsiE. https:// drive.google.com/file/d/oBxBJy7UhDt22SVpMRjluRHhDSFU/view.

Esteban Frades, S. (20I4) La Inspección de Educación. Historia, pensamiento y vida. Oviedo: ADIDE Castilla y León y KRK Ediciones.

Gil de ZÁrate, A. (1995) De la Instrucción pública en España, tomo i. Oviedo: Pentalfa (Edición facsímil del original del año i855. Madrid: Imprenta del Colegio de Sordo-mudos).

Sarasúa, A. (20Io) La Inspección de educación en vía muerta. Revista Escuela, núm. 3875.

Secadura, T. (20II) El referente de la Inspección educativa: el centro docente versus el sistema educativo. Avances en Supervisión Educativa, núm. I5. https://avances.adide.org/index. $\mathrm{php} / \mathrm{ase} / \mathrm{article} / \mathrm{view} / 485 / 326$.

Soler Fiérrez, E. (2015) Decálogo de las competencias profesionales del inspector de Educación. REICE, Revista Iberoamericana sobre Calidad, Eficacia y Cambio en Educación, I3 (4), I49-I60. http://www.rinace.net/reice/numeros/arts/voliznum4/art9.htm. 
